\title{
Utilization of MASH Planetary Nebula Catalogue of the Year 2006 for the Determination of the Milky-Way Plane
}

\author{
A.M. Sendi \\ M.Sc. Student, Astronomy and Space Science, \\ Faculty of Science, King Abdulaziz University, \\ Jeddah, Saudi Arabia
}

\begin{abstract}
In this paper, the equatorial coordinates of the galactic pole $\left(\alpha_{p}, \delta_{p}\right)$ for the epoch 2000.0. was computed using the most recent data of the MASH planetary nebulae catalogue of the year 2006. As a result, the inclination of the Milky way plane to the celestial equator $i_{g}$ and the right ascension of its ascending node $\Omega_{\mathrm{g}}$ at the same epoch are also computed.
\end{abstract}

Keywords: Position astronomy, Galactic structure, ephemeris.

\section{Introduction}

In many applications of position astronomy and galactic structure, e.g. ${ }^{[1]}$, the determination of the orientation of the Milky way plane's to equatorial plane at certain epoch is of extreme importance, not only to coordinate transformations, but also for some problems concerning the relation between the astrophysical properties of celestial objects with their condensation towards a plane, the Milky way (e.g. the Milky way condensation of young Population I objects). Recently, a general analytical method was developed ${ }^{[2]}$, [hereafter will be referred to as Paper I] for the determination of any celestial plane orientation of objects with respect to fundamental plane at certain epoch.

In the present paper, this method is applied for the determination of: 1. the equatorial coordinates of the galactic pole $\left(\alpha_{p}, \delta_{p}\right)$ for the epoch 2000.0. 2. The inclination of the Milky way plane's to the celestial 
equator $i_{g}$ and the right ascension of its ascending node $\Omega_{\mathrm{g}}$ at the same epoch. The input data of this application is MASH planetary nebulae catalogue (Macquarie/AAO/Strasbourg Halpha Planetary Nebula Catalogue) of the year $2006^{[3]}$. In this catalogue over 900 true, likely and possible new Galactic planetary nebulae (PNe) are listed.

\section{Formulations}

According to Paper I, the galactic constants $\left(\alpha_{p}, \delta_{p}, i_{g}, \Omega_{g}\right)$ for the epoch 2000.0 could be determined from:

$$
\delta_{\mathrm{p}}=\sin ^{-1}(\mathrm{n}), \alpha_{\mathrm{p}}=\tan ^{-1}\left(\frac{\mathrm{m}}{\ell}\right), \mathrm{i}_{\mathrm{g}}=90^{\circ}-\delta_{\mathrm{p}} \text { and } \Omega_{\mathrm{g}}=90^{\circ}+\alpha_{\mathrm{p}}
$$

where $(\lambda, \ell, \mathrm{m}, \mathrm{n})$ are the global minimum of the function:

$$
\mathrm{F}(\ell, \mathrm{m}, \mathrm{n}, \lambda)=\mathbf{L}^{\mathrm{T}} \mathbf{A} \mathbf{L}-\lambda\left(\mathbf{L}^{\mathrm{T}} \mathbf{L}-1\right),
$$

$\mathbf{L} \equiv(\ell, \mathrm{m}, \mathrm{n})$ and $\lambda$ are respectively the eigen vectors and the eigen values of the symmetric matrix $\mathbf{A}_{3 \times 3}\left[a_{i j}\right]$ :

$$
\begin{gathered}
\mathrm{a}_{11}=\sum_{\mathrm{i}=1}^{\mathrm{N}} \mathrm{x}_{\mathrm{i}}^{2} ; \mathrm{a}_{12}=\sum_{\mathrm{i}=1}^{\mathrm{N}} \mathrm{x}_{\mathrm{i}} \mathrm{y}_{i} ; \mathrm{a}_{13}=\sum_{\mathrm{i}=1}^{\mathrm{N}} \mathrm{x}_{\mathrm{i}} \mathrm{z}_{i} ; \mathrm{a}_{22}=\sum_{\mathrm{i}=1}^{\mathrm{N}} \mathrm{y}_{\mathrm{i}}^{2} ; \\
\mathrm{a}_{23}=\sum_{\mathrm{i}=1}^{\mathrm{N}} \mathrm{y}_{\mathrm{i}} \mathrm{z}_{i} ; \mathrm{a}_{33}=\sum_{\mathrm{i}=1}^{\mathrm{N}} \mathrm{z}_{\mathrm{i}}^{2} .
\end{gathered}
$$

The coordinates $\left(\mathrm{x}_{\mathrm{i}}, \mathrm{y}_{\mathrm{i}}, \mathrm{z}_{\mathrm{i}}\right) ; \mathrm{i}=1,2, \cdots, \mathrm{N}$ of the $\mathrm{N}$ selected PNe at unit distance from the sun are:

$$
\mathrm{x}_{\mathrm{i}}=\cos \delta_{\mathrm{i}} \cos \alpha_{\mathrm{i}} \quad ; \quad \mathrm{y}_{\mathrm{i}}=\cos \delta_{\mathrm{i}} \sin \alpha_{\mathrm{i}} \quad ; \quad \mathrm{z}_{\mathrm{i}}=\sin \delta_{\mathrm{i}}
$$

where $\left(\alpha_{i}, \delta_{i}\right)$ are the equatorial coordinates of the nebulae for the epoch 2000.0. 


\section{Applications}

\subsection{Data}

The equatorial coordinates of PNe for the epoch 2000.0 are taken from Hipparcos main catalogue whose table number at CDS (Strasbourg astronomical data center), is: V/127 MASH catalogue planetary nebulae ${ }^{[3]}$.

\subsection{Numerical Results}

The results $\left(\alpha_{\mathrm{p}}, \delta_{\mathrm{p}}, \mathrm{i}_{\mathrm{g}}, \Omega_{\mathrm{g}}\right)$ for the epoch 2000.0 are given below. Each of these results is the average of five values corresponding to galactic latitudes $b=1^{\circ}, 2^{\circ}, 3^{\circ}, 4^{\circ}, 5^{\circ}$.

$$
\alpha_{\mathrm{p}}=192.833^{\circ}: \delta_{\mathrm{p}}=26.9014^{\circ} ; \mathrm{i}_{\mathrm{g}}=63.0986^{\circ} ; \Omega_{\mathrm{g}}=282.833^{\circ}
$$

In concluding this paper, general analytical method ${ }^{[2]}$ for the determination of any celestial plane orientation of objects with respect to fundamental plane at certain epoch was applied for the determination of: 1. the equatorial coordinates of the galactic pole $\left(\alpha_{p}, \delta_{p}\right)$ for the epoch 2000.0. 2. The inclination of the Milky way plane to the celestial equator $\mathrm{i}_{\mathrm{g}}$ and the right ascension of its ascending node $\Omega_{\mathrm{g}}$ at the same epoch. The input data of this application is MASH planetary nebulae catalogue of the year $2006^{[3]}$.

\section{References}

[1] Meeus, J., Astronomical Alogorithms, Willmann-Bell, Inc. (1991).

[2] Sharaf, M.A., Journal of Romanian Astronomical Journal (to appear) (2007).

[3] Parker, Q.A., Acker, A., Frew, D.J., Hartley, M., Peyaud, A.E.J., Phillipps, S., Russeil D., Beaulieu, S.F., Cohen, M., Koppen, J., Marcout, J., Miszalski, B., Morgan, D.H., Morris, R.A.H., Ochsenbein, F., Pierce, M.J. and Vaughan, A.E., Mon. Not. R. Astrono. Soc., 373(1): 79-94 (2006). 


\title{
استخدام كتالوج MASH للسدم الكوكبية لعام 7 . . זم لتعبين مستوى الطريق اللبني
}

\author{
أحلام محمد سندي \\ طالبة ماجستبر - فلك و علوم فضاء

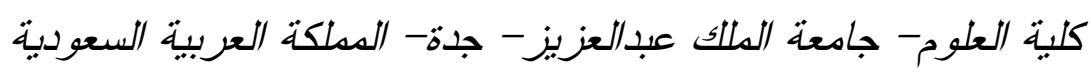

المستخلص. تم في هذه البحث تطويع طريقة تحليلية عامة لتعيين الإحداثيات الاستو ائية لقطب المجرة للحقبة 2000.0 و أيضًا تعيين ميل الطريق اللبني على الاستو اء السماوي، وطول عقدة الصعود له له لهابله

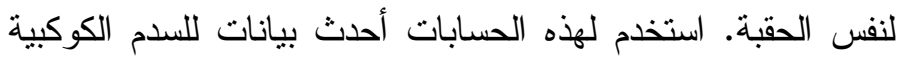
المنشورة في كتالوج 2006 MASH. 